\title{
Selection of Energy Efficient Routing Protocol for Irrigation Enabled by Wireless Sensor Networks
}

\author{
Zainal Abedin ${ }^{1}$, Sukanta Paul ${ }^{1}$, Sharmin Akhter ${ }^{1}$, Kazy Noor-e-Alam Siddiquee ${ }^{1}$, \\ Mohammad Shahadat Hossain ${ }^{2}$, and Karl Andersson ${ }^{3}$ \\ ${ }^{1}$ Faculty of Science, Engineering and Technology, University of Science \& Technology Chittagong-1079, Bangladesh \\ ${ }^{2}$ Department of Computer Science and Engineering, University of Chittagong-4331, Bangladesh \\ ${ }^{3}$ Pervasive and Mobile Computing Laboratory, Luleå University of Technology, SE-931 87 Skellefteå, Sweden
}

jakcse99@gmail.com,skpaulustc@gmail.com,sarak_20@yahoo.com, knas11@gmail.com, hossain_ms@cu.ac.bd, karl.andersson@1tu.se

\begin{abstract}
Wireless Sensor Networks (WSNs) are playing remarkable contribution in real time decision making by actuating the surroundings of environment. As a consequence, the contemporary agriculture is now using WSNs technology for better crop production, such as irrigation scheduling based on moisture level data sensed by the sensors. Since WSNs are deployed in constraints environment, the life time of sensors is very crucial for normal operation of the networks. In this regard routing protocol is a prime factor for the prolonged life time of sensors. This research focuses the performances analysis of some clustering based routing protocols to select the best routing protocol. Four algorithms are considered, namely Low Energy Adaptive Clustering Hierarchy (LEACH), Threshold Sensitive Energy Efficient sensor Network (TEEN), Stable Election Protocol (SEP) and Energy Aware Multi Hop Multi Path (EAMMH).The simulation is carried out in Matlab framework by using the mathematical models of those algorithms in heterogeneous environment. The performance metrics which are considered are stability period, network lifetime, number of dead nodes per round, number of cluster heads $(\mathrm{CH})$ per round, throughput and average residual energy of node. The experimental results illustrate that TEEN provides greater stable region and lifetime than others while SEP ensures more throughput.
\end{abstract}

Keywords-Routing Protocol, Life Time, LEACH, TEEN, SEP, EAMMAH, Heterogeneous, Stable Region, Throughput

\section{INTRODUCTION}

WSNs are promising and revolutionary technology where machines and the environment are integrated to provide numerous applications such as agriculture, surveillance, environment control, intelligent buildings, facility management, health care and logistics.

A sensor node is typically capable of sensing environmental parameters such as moisture level, temperature, humidity due to the processing and communication capabilities. WSNs are formed using these sorts of sensor nodes. However, the sensor nodes have limited energy as they are powered by tiny battery. As long as the nodes are alive, the WSNs can sustain for normal operation. Hence, energy saving is very crucial and challenging issue for sensor nodes. For large scale network it concerns more importance because the sensors in this sort of networks participate in more traffic and hence, reduce the energy quickly. To save the energy, different sorts of algorithms have been developed in MAC layer and network layer [1]. To manage and ensure prolonged life time of WSNs, many protocols have been presented in $[2][3][4][5][6][7][8][9][10][11][12][13][14][15]$. Most of them are based on the hierarchical relationship because of their robustness in scalibility and power consumtion for large scale networks. This kind of routing protocols can be classified into two classes namely cluster based and tree based structure. In cluster based protocols the nodes form cluster with one cluster head $(\mathrm{CH})$. All the nodes in a cluster send the data to the $\mathrm{CH}$ and then $\mathrm{CH}$ forward it to the sink node. In tree based all the nodes send their packet to the root of the tree through intermediate node and the root forwards it to central node. In this regard, the contribution of this paper is to select an energy efficient clustering based routing protocol in the case of agriculture for irrigation scheduling.

WSNs are becoming emerging technology in modern agriculture for smart irrigation, harvesting, seeding and crop monitoring etc. Water and energy is very burning issue for the sustainable development. The usage of water and energy can be optimized by taking real time decision of irrigation scheduling using moisture level data of the field. To set up a smart irrigation we need soil moisture sensor, sink node and a computing system as backend. The normal operation of such type of system depends on the lifetime of the network. One of the important parameter for less power consumption is the selection of optimum routing protocol. Another important factor is deployment of the nodes, whether it is random or deterministic. The network topology and data aggregation methods are also responsible for power consumption. All these factors are related to the network design of WSNs. The robustness of these sorts of network depends on the lifetime of sensors and the data rate or packet loss ratio. To develop a satisfying sensor enabled irrigation scheduling system, the adaptation of these parameters draws significant design issues [16][17].

The structure of this paper is as follows: Section 2 describes the radio model and analytical models of LEACH, SEP, TEEN and EAMMH routing protocols. Section 3 describes 
the environment of simulation setup. Section 4 shows the experimental results for performance evaluation. Finally, Section 5 summarizes our conclusion and future work.

\section{ENERGY MODEL AND RoUting PROTOCOLS}

\section{A. Radio Energy Consumption Model}

In this simulation, to calculate the energy consumption of the trasmitter and receiver the following radio energy model is adopted [18]. In order to achieve an acceptable Signal to Noise Ratio (SNR) in transmitting an $L$ bit messages over a distance $(d)$, the energy consumption by the radio is given by:

$E_{T x}(L, d)=\left\{\begin{array}{c}L * E_{\text {elec }}+L * \epsilon_{f s} * d^{2}, \text { if } d \leq d_{0} \\ L * E_{\text {elec }}+L * \epsilon_{m p} * d^{24}, \text { if } d>d_{0}\end{array}\right.$

$E_{T x}(L, d)=L * E_{\text {elec }}$

where $E_{\text {elec }}$ is the energy dissipation per bit for transmitter or receiver, $\varepsilon_{f s}$ is the free space amplification parameter, $\varepsilon_{m p}$ is the multi path fading signal amplification coefficient; $d$ is the distance between source and destination, $L$ is the size of message in bits, and $d_{0}=\sqrt{\frac{\epsilon_{f s}}{\epsilon_{m p}}}$

\section{B. Low Energy Adaptive Clustering Hierarchy (LEACH)}

LEACH is hierarchical routing protocol which conserves energy of the sensors by dynamic cluster formation. A Cluster head is elected in a random manner at the beginning of each round. All the nodes send their data to the base station via the $\mathrm{CH}$. Every round, LEACH executes two phases: 1) the set up phase for cluster formation, and 2) the steady state for data transmission. In the set up phase, each node calculates the probability based on the residual energy to participate in cluster election. The node with the highest probability is elected as a $\mathrm{CH}$. After the election process, the $\mathrm{CH}$ broadcasts a message using the CSMA media access protocol. All the normal nodes select their $\mathrm{CH}$ using the signal strength of the received messages. Then every normal node sends an acknowledgment to its $\mathrm{CH}$. The $\mathrm{CH}$ sets up a TDMS scheduling for its members for data communication. In LEACH, an optimal percentage of sensors has to elected as $\mathrm{CH}$ in every round under the assumption of uniform distribution of the nodes. Initially each node can become a cluster head with an optimal probability. A sensor node can be $\mathrm{CH}$ once per epock. After each round the probability of nodes of non elected nodes $(G)$ is increased to become a $\mathrm{CH}$ in the same epock. In each round the nodes of $\mathrm{G}$ choose a random number in the interval of [0 1]. If the random is bigger than a threshold value $T(\mathrm{~s})$, then the node can be elected as a $\mathrm{CH}$ in current iteration. The threshold is calculated using the equation 3 . In the steady state, the nodes send their data frame to the $\mathrm{CH}$ by using TDMD multiple access mechanism to avoid collision. The $\mathrm{CH}$ aggregates the received frame and discards the similar frame which reduces the traffic cost as well as save energy. In this simulation, the heterogeneity environment is considered to evaluate the behavior of LEACH. Initially, All nodes have some initial energy in homogeneous network while some percentages of node are set to as advance node in heterogeneous network where the energy of this advanced node is set to (initial energy $\times(1+\alpha)$ ) as well as energy of normal nodes is set to initial energy, randomly. The threshold is calculated based on the following formula:

$T(s)= \begin{cases}\frac{p_{\text {opt }}}{1-p_{\text {opt }}\left(\text { r mod } \frac{1}{p_{\text {opt }}}\right)} & , \text { if } s \varepsilon G \\ 0 & , \text { otherwise }\end{cases}$

where $r$ is the round number and $p_{\text {opt }}$ is the initial optimal probability that is calculated by using Eqs (4) and (5).

$$
\begin{aligned}
& \mathrm{p}_{\mathrm{opt} t}=\frac{k_{o p t}}{n} \\
& k_{\text {op } t}=\sqrt{\frac{n}{2 \pi}} \sqrt{\frac{\epsilon_{f s}}{\epsilon_{m p}}} \frac{M}{d^{2}{ }_{\text {toBS }}}
\end{aligned}
$$

\section{Stable Election Protocol (SEP)}

SEP is a heterogeneous hierarchical clustering routing protocol. The distribution of energy in classical routing protocol is even whereas in SEP the energy distribution is not equal. In SEP some nodes are given more energy than the others, called advanced node and the rest of nodes are called normal nodes. SEP was proposed to prolong the stability period of the networks. Because of heterogeneity the election probability of nodes are weighted and this feature of SEP increases the stability period. The weighted probabilities of normal and advanced nodes are determined by using Eqs (6) and (7).

$$
\begin{aligned}
& p_{\text {nrm }}=\frac{p_{\text {opt }}}{1+\alpha * m} \\
& p_{\text {adv }}=\frac{p_{\text {opt }}}{1+\alpha * m} *(1+\alpha)
\end{aligned}
$$

The threshold for becoming the cluster head is calculated by using the following equations:

$$
\begin{gathered}
T\left(s_{n r m}\right)=\left\{\begin{array}{lc}
\frac{p_{n r m}}{1-p_{n r m}\left(r \text { mod } \frac{1}{p_{n r m}}\right)} & , \text { if } s_{n r m} \in G_{n r m} \\
0 & , \text { otherwise }
\end{array}\right. \\
T\left(s_{\text {adv }}\right)=\left\{\begin{array}{lc}
\frac{p_{a d v}}{1-p_{a d v}\left(r \text { mod } \frac{1}{p_{a d v}}\right)} & , \text { if } s_{a d v} \in G_{a d v} \\
0 & , \text { otherwise }
\end{array}\right.
\end{gathered}
$$

where, $G_{\text {nrm }}$ is the set of normal nodes and $G_{\text {adv }}$ is the set of advanced nodes

\section{Threshold Sensitive Energy Efficient Sensor Network (TEEN)}

TEEN is a reactive and even driven multi level hierarchical clustering protocol. In TEEN the sensors turn on the radio for transmission of data when the sensed data changes significantly. Hence, no periodic transmission is carried out. The cluster is formed with a $\mathrm{CH}$ taking the nodes in closer distance where every node becomes $\mathrm{CH}$ in turns. Every node in a cluster is elected as $\mathrm{CH}$ for a time interval called cluster period. This process continues until sink node is reached. The node transmits data based on a threshold parameter. After the cluster formation, the $\mathrm{CH}$ broadcasts the threshold values to its members. Unless the sensed data cross the threshold value, the node is not triggered to turn on radio. Two types of threshold are used in TEEN protocol namely hard threshold and soft threshold. The hard threshold limits the range of the sensed value of the node and the soft threshold defines the changes. Both these threshold parameters limit the unnecessary transmission. Hence, the energy consumption in this protocol is less than proactive method. As a result the lifetime of the network is increased. In TEEN, lower level CH receives and aggregates the data from the members of the 
cluster and forwards it to higher level $\mathrm{CH}$. In this way, the hierarchy relation is formed in this algorithm. This protocol is not well suited for application where the system needs continuous data streaming.

\section{E. Energy Aware Multi-Hop Multi-Path Hierarchy (EAMMH)}

This is reactive multi path hierarchical routing protocol. Here, cluster head election method is similar to LEACH protocol. The $\mathrm{CH}$ maintains a routing table for multi hop routing for the cluster members. To choose an optimal path from its routing table entries, a heuristic function is used and the heuristic function is given by:

$$
h=K\left(E_{\text {avg }} / h_{\min } * t\right)
$$

where $K$ is a constant, $E_{\text {avg:is the average energy of the current }}$ path, $h_{\min }$ the minimum hop count in the current path, and $t$ the traffic of the path. The path with highest heuristic value is chosen. When minimum energy of this path is greater than a threshold, the path will be elected as an optimum routing path. Else the path with the next highest heuristic value is chosen. The minimum energy of a path is evaluated by using Eq. (11).

$$
E_{\min }=E_{\text {avg }} / \text { const }
$$

where const is a constant parameter. If there is no node which has $E_{\min }$ greater than the threshold energy, the node with highest minimum energy is selected. Periodic updates of the information about paths and routing table entries are done at regular intervals to keep the network stable.

\section{SimUlation ENVIRONMENT}

To compare the performances of hierarchical routing protocols in heterogeneous scenarios, simulations were performed in a MATLAB environment. The mathematical model of each protocol is considered to conduct the simulation. The radio features of this simulation are outlined in the Table1. The deployment of the sensors is implemented in random manner as shown in Fig. 1. In Fig. 2 dynamic cluster formation of SEP protocol is depicted. The placement of the sink node (Base Station) is at the centre of the network area. This simulation addresses the heterogeneity feature of the sensor networks to figure out real scenarios. To introduce this heterogeneity, a percentage of the sensors are energized with more energy than rest of the sensors of the network. Two heterogeneous scenarios are considered where the percentages of advanced nodes $(\mathrm{m})$ are set to 0.2 and 0.5 respectively. The optimum initial probability $\left(p_{\text {opt }}\right)$ is assumed to be 0.1 for simplicity and the extra energy factor $(\alpha)$ between simple and advanced is assumed to be 1.0.

TABLE 1 SIMULATION PARAMETERS

\begin{tabular}{|l|c|}
\hline Parameter & Value \\
\hline Transmit/Receive Energy & $E_{\mathrm{elc}}=50 \mathrm{~nJ} / \mathrm{bit}$ \\
\hline Data Accumulation & $E_{\mathrm{DA}}=5 \mathrm{~nJ} / \mathrm{bit}$ \\
\hline Transmitter Amplification $\left(d<=d_{\mathrm{o}}\right)$ & $E_{\mathrm{fs}}=10 \mathrm{pJ} / \mathrm{bit} / \mathrm{m}^{2}$ \\
\hline Transmitter Amplification $\left(d<=d_{\mathrm{o}}\right)$ & $E_{\mathrm{mp}}=0.0013 \mathrm{pJ} / \mathrm{bit}^{4} \mathrm{~m}^{4}$ \\
\hline Initial energy & $0.5 \mathrm{~J}$ \\
\hline Packet size & $4000 \mathrm{bits}$ \\
\hline Network Dimensions & $100 \times 100,200 \times 200$ \\
\hline
\end{tabular}

\begin{tabular}{|l|c|}
\hline Node Density $(n)$ & 100,200 \\
\hline Percentages of advanced nodes $(m)$ & $0.2,0.5$ \\
\hline Scenario 1 & $100 \times 100, n=100, m=0.2$ \\
\hline Scenario 2 & $100 \times 100, n=200, m=0.2$ \\
\hline Scenario 3 & $100 \times 100, n=100, m=0.5$ \\
\hline Scenario 4 & $200 \times 200, n=100, m=0.2$ \\
\hline
\end{tabular}

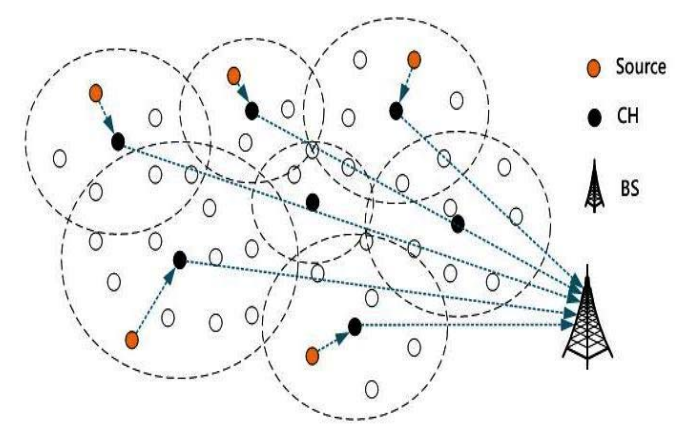

Fig. 1. Cluster Based Sensor Networks

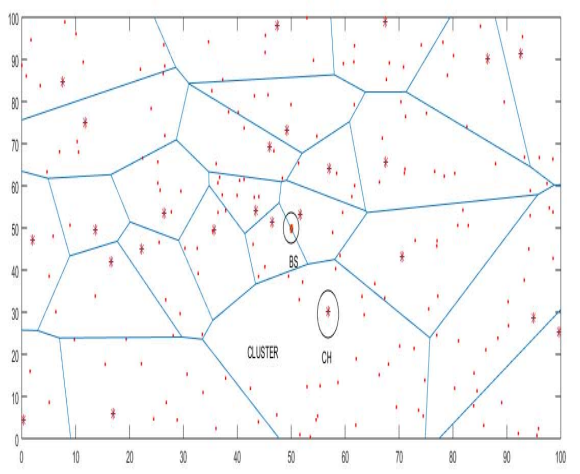

Fig. 2. Dynamic Cluster Formation (SEP)

\section{Simulation Results}

To evaluate the performances of the clustering routing protocols LEACH, SEP, TEEN, and EAMMH in different scenarios, the following parameters were taken into consideration. This simulation also makes a comparison between LEACH, Direct Transmission (DT), and Minimum Transmission Energy (MTE).

- Stability period: it is the time duration from the beginning of the network until the death of first sensor node. It is called stable region.

- Instability period: this is a measure of time between the death of first sensor node and the death of last node. It is referred as unstable region.

- Network Life Time: the duration from the beginning of the network until the death of last sensor node.

- Number of Cluster Heads: This reflect the number of nodes which receive, aggregate and forward data frame to the sink.

- Number of Dead Nodes: the nodes which finished their energy. 
- Throughput: the rate of data sent from the $\mathrm{CHs}$ to the sink.

- Residual energy of nodes

Direct transmission of a packet to the base station is the simplest routing method for small scale networks. However, the weakness of such a protocol is that if the nodes are deployed at larger distance from the BS, the energy consumption will be more. Also packet forwarding for longer distance may be lost due to fading and collision. Whereas in minimum transmission energy method, data is transmitted using the shortest path in terms of energy. The nodes between the sender and BS act as relays and these relay nodes die quickly because of tunneling more traffic. So, in the both approaches the energy is not uniformly distributed which reduces the lifetime of the network. Also, in these methods, some of the sensors are not monitored for a significant time. As a result, the sensing process is not properly achieved. To address these sorts of problem LEACH clustering protocol was proposed where the energy is distributed evenly by the dynamic selection of cluster head. The experimental result of Fig. 3 illustrates that the lifetime of the network in case of LEACH protocol is much better than DT and MTE protocols.

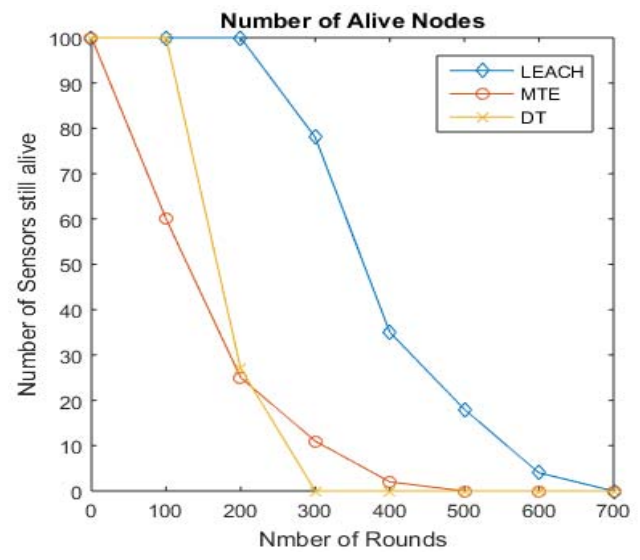

Fig. 3. Number of Alive Nodes

The simulation statistics from Tables 2 to 4 depict stable and instable region of the selected routing protocols in terms of time instant (rounds). Table 2 represents that the TEEN protocol maintains superior stable region. The reason is that in TEEN protocol data transmission is dependent on the soft and hard threshold. These two parameters limit the transmission significantly. This table also shows that SEP has better stable region than basic LEACH protocol in heterogamous scenario due to the weighted cluster election probability where some nodes are equipped extra energy. From Table 3 we observe that the SEP protocol holds less instable region in all scenarios. The Table 4 interprets the life time of the network for the four protocols where TEEN surpasses the others. Considering the network lifetime and stability it is inferred that TEEN has the best performances and prolong the lifetime of WSNs.

TABLE 1 Stable Region (Round)

\begin{tabular}{|l|l|l|l|l|}
\hline Protocol & Scenario1 & Scenario2 & Scenario3 & Scenario4 \\
\hline LEACH & 613 & 590 & 630 & 250 \\
\hline SEP & 1126 & 1119 & 1283 & 760 \\
\hline TEEN & $\mathbf{1 4 3 3}$ & $\mathbf{1 4 3 1}$ & $\mathbf{1 5 3 5}$ & $\mathbf{1 3 3 1}$ \\
\hline EAMMH & 583 & 556 & 610 & 311 \\
\hline
\end{tabular}

TABLE 2 Unstable region (Round)

\begin{tabular}{|l|l|l|l|}
\hline Protocols & Scenario 1 & Scenario 2 & Scenario 3 \\
\hline LEACH & $4385+$ & 3095 & 1897 \\
\hline SEP & $\mathbf{2 4 2 2}$ & $\mathbf{2 8 3 9}$ & $\mathbf{2 3 6 4}$ \\
\hline TEEN & $4565+$ & 3568 & 3465 \\
\hline EAMMH & 2794 & $4444+$ & 2701 \\
\hline
\end{tabular}

TABLE 3 Network life time (Round)

\begin{tabular}{|c|c|c|c|}
\hline Protocols & Scenario 1 & Scenario 2 & Scenario 3 \\
\hline LEACH & 2528 & 3684 & $5000+$ \\
\hline SEP & 3649 & 3959 & 3550 \\
\hline TEEN & $\mathbf{5 0 0 0 +}$ & $\mathbf{5 0 0 0 +}$ & $\mathbf{5 0 0 0 +}$ \\
\hline EAMMH & 3312 & $5000+$ & 3379 \\
\hline
\end{tabular}

Figures 4 to 7 illustrate the behavior of number of dead nodes per round for the protocols in different cases. Form these figures it is observed that TEEN. This is because of the prolonged stable period of the TEEN protocol.

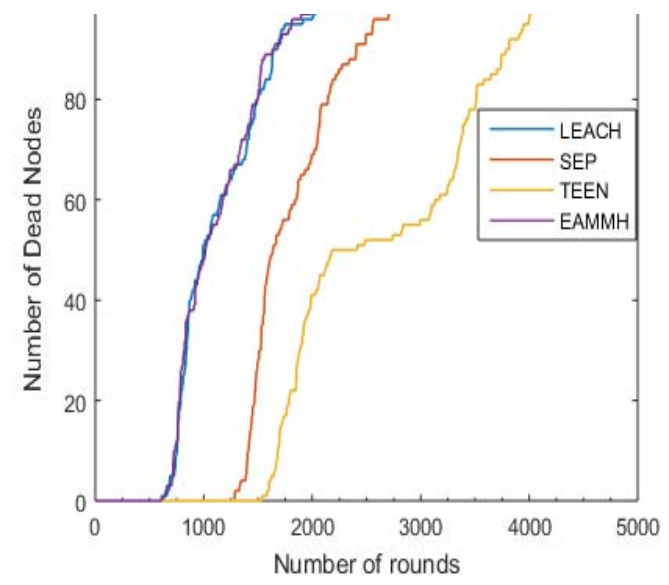

Fig. 4. Number of Dead Nodes (Scenario 1)

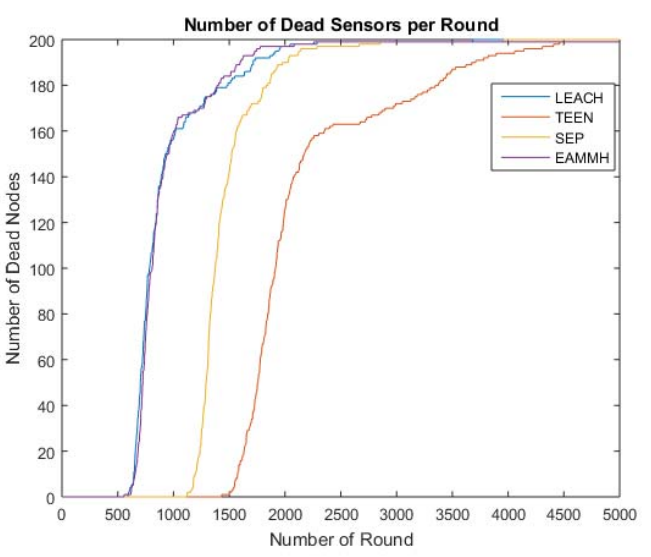

Fig. 5. Number of Dead Nodes (Scenario 2) 


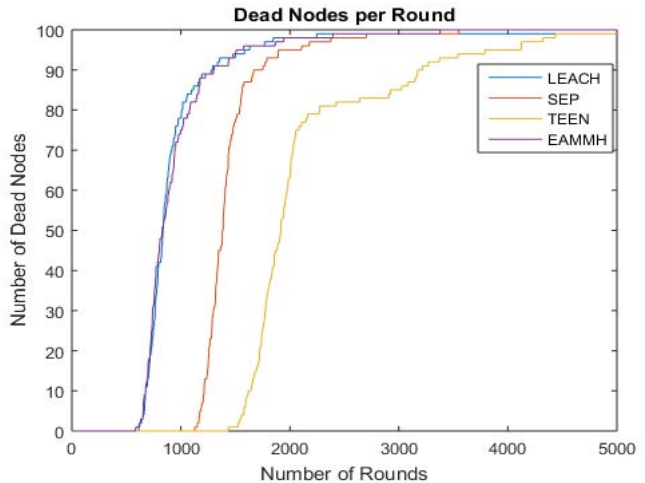

Fig. 6. Number of Dead Nodes (Scenario 3)

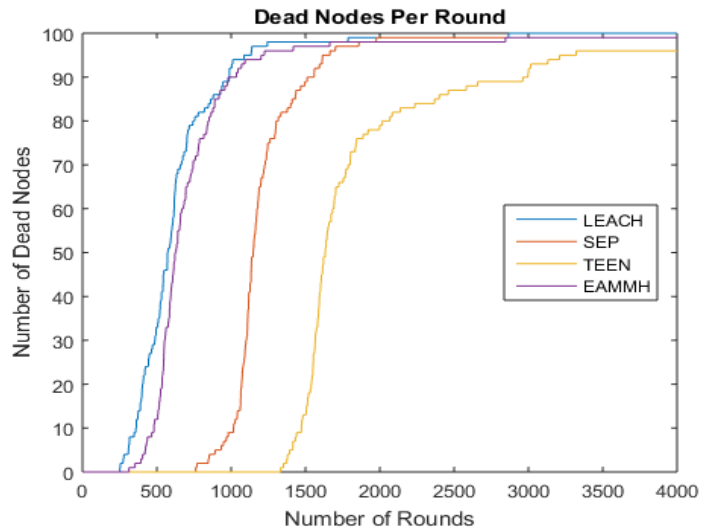

Fig. 7. Number of Dead Nodes Life Time (Scenario 4)

Throughput is also an important factor to evaluate robustness of routing protocol. Receiving high data at the BS means that the routing protocol is performing well. Figures 8 to 10 illustrate the comparative picture of throughput for the protocols for two scenarios. The comparison shows that SEP performs better throughput by sending more packet to the base station. Since SEP protocol elects more number of cluster head due to the heterogeneity and weighted probability calculation of the advanced and normal nodes.

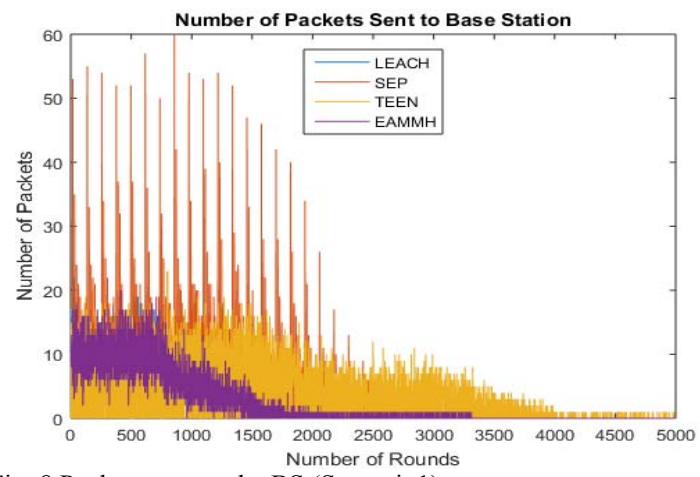

Fig. 8 Packets sent to the BS (Scenario1)

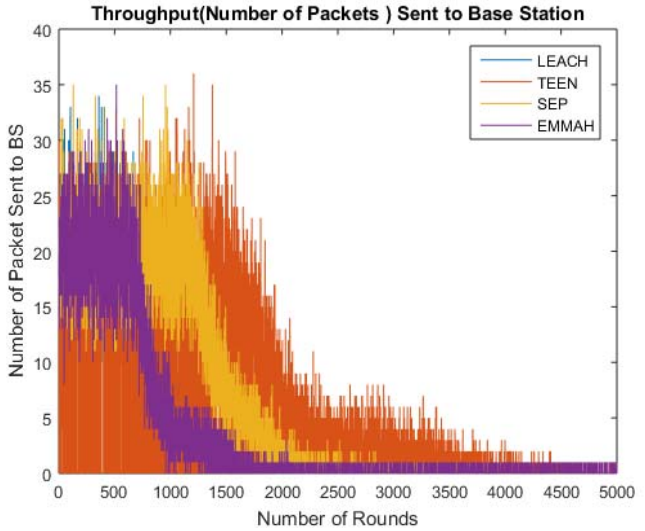

Fig. 9. Packets sent to the BS (Scenario 2)

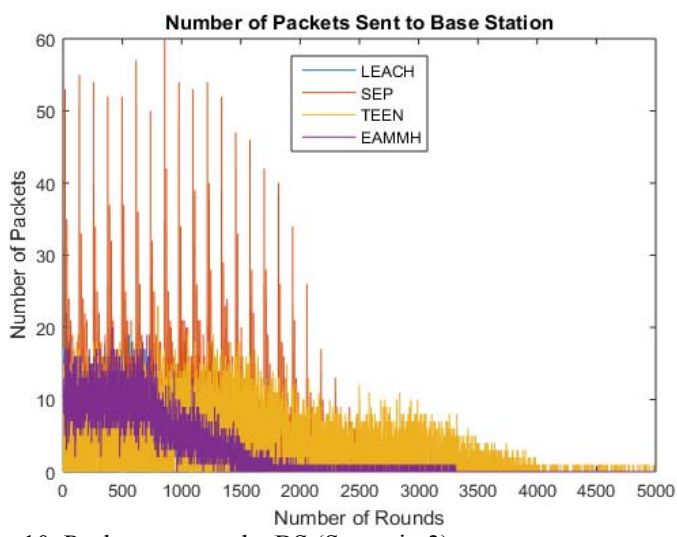

Fig. 10. Packets sent to the BS (Scenario 3)

Figures 11,12 and 13 describe the behavior of LEACH, TEEM, SEP and EAMMH in regards of number of cluster heads per round for different scenarios. The scenarios present that more number of cluster heads is elected for SEP protocol till an upper bound, because the election probability calculation is weighted among the normal and advanced nodes. In LEACH, fewer cluster heads are elected due to unstable cluster formation.

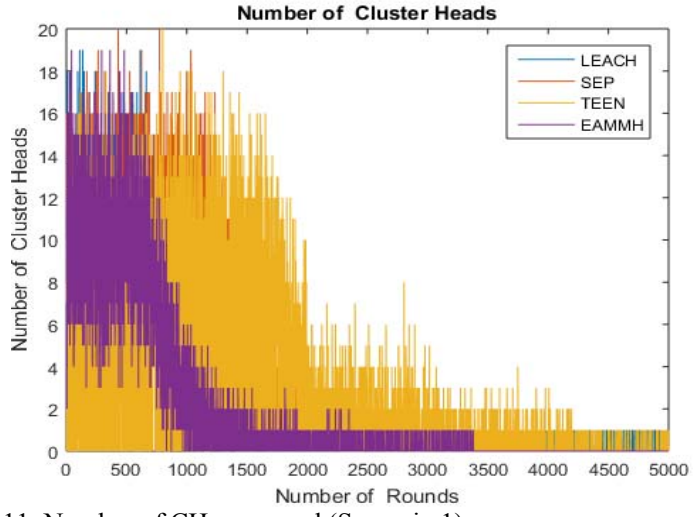

Fig. 11. Number of $\mathrm{CH}$ per round (Scenario 1) 


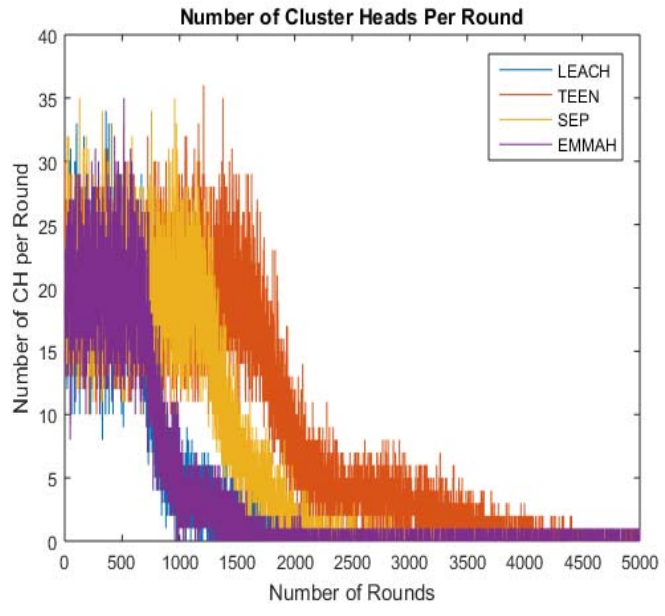

Fig. 12. Number of $\mathrm{CH}$ per round (Scenario 2)

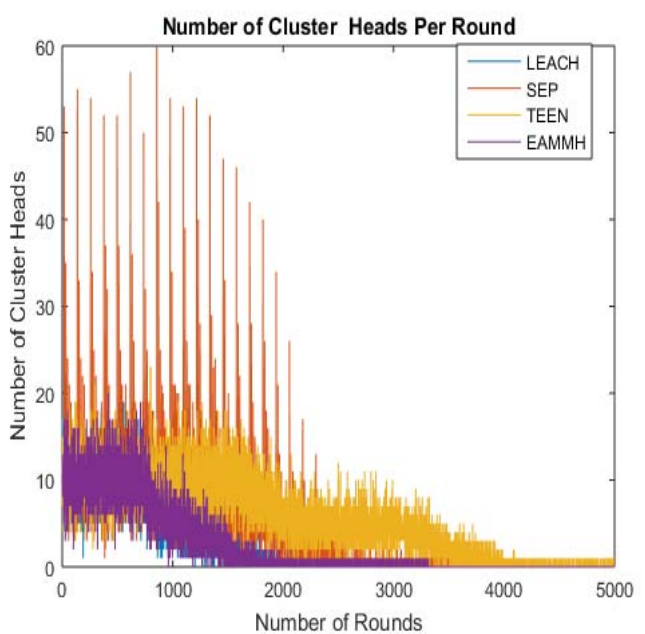

Fig. 13. Number of $\mathrm{CH}$ per round (Scenario 3)

Residual energy of the sensor is a crucial parameter to enhance the lifetime of sensor networks. The lower energy consumption ensure the prolonged life time of the sensor nodes. Figures 14 to 17 depict the residual average energy of node in each round for the selected clustering protocols in different scenarios. These figures illustrate that the average residual energy of the node is better in TEEN hierarchical routing protocol than the others due to its lower data transmission rate to the sink.

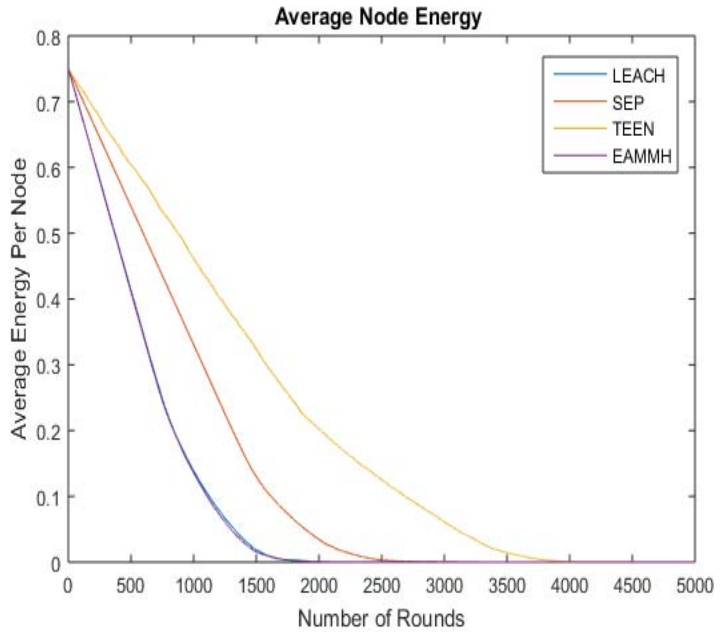

Fig.14. Residual energy (Scenario 1)

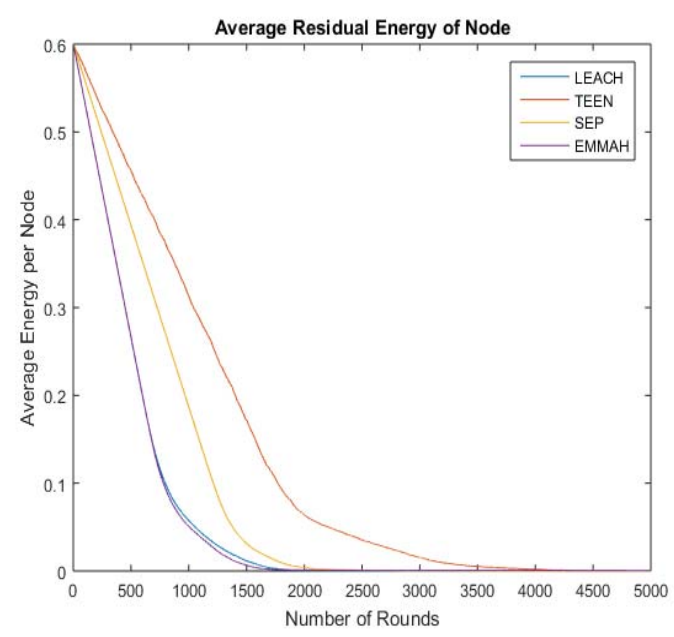

Fig. 15. Residual energy (Scenario 2)

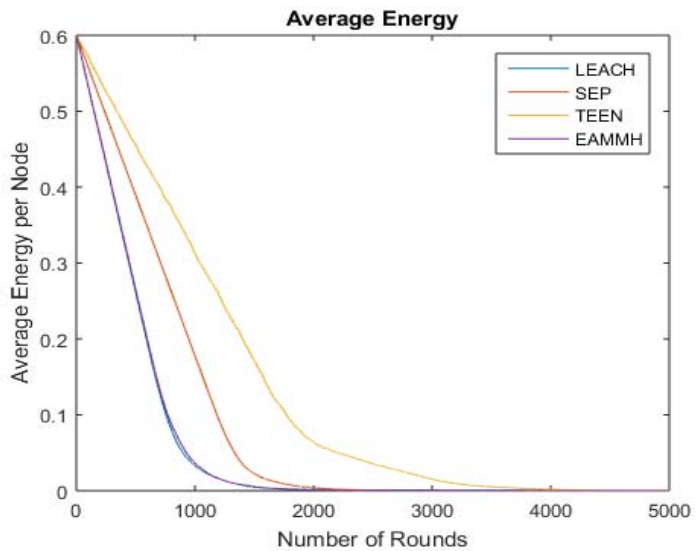

Fig. 16. Residual energy (Scenario 3) 


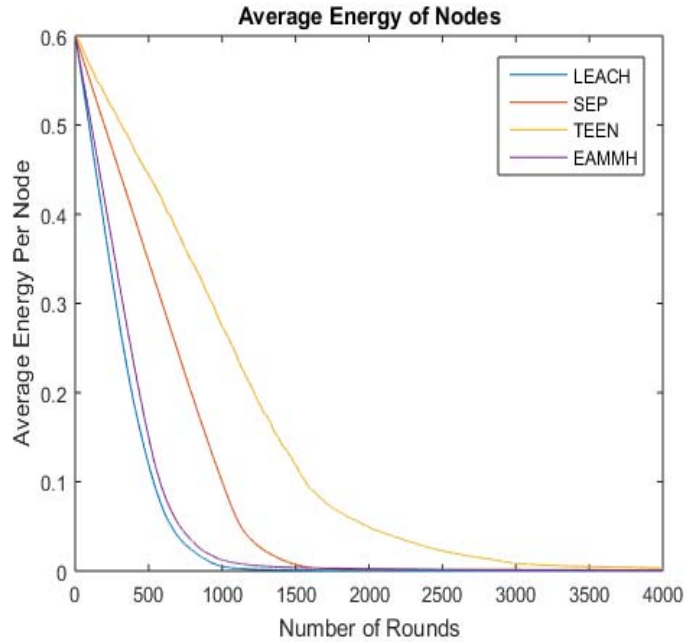

Fig. 17. Residual energy (Scenario 4)

\section{CONCLUSION AND FUTURE WORK}

The application areas of WSNs are diverse and are increasing day by day. The prime challenge in sensor networks is the minimum energy consumption while keeping the network alive in operation. In this regard, routing protocol is a cardinal and necessary part for the design of WSNs. The design of WSNs is also based on application specific requirements. The objective of this research is to compare the performances of some hierarchical clustering routing protocols (LEACH, TEEN, SEP, and EAMMH) for the development of irrigation scheduling based on soil moisture sensor nodes. The goal of all hierarchical routing algorithms is to prolong the network longevity by using optimum energy utilization. The parameters which are selected for comparisons are stable region, lifetime, number of dead nodes, number of cluster heads, throughput and residual energy of the nodes and the environment is heterogeneous. The simulations were performed in a Matlab environment by using theoretical models of the protocols. The simulation output implies that the stable region and lifetime of the TEEN protocol is better than the others along with the throughput of the SEP protocol, which is the best for an upper bound.

Our future work will be to make analytical comparison between clustering and tree based routing protocols and set up a test bed for real time performance analysis.

\section{ACKNOWLEDGEMENT}

We would like to extend our gratitude to the Swedish Research Council for the funding (under grant 2014-4251) this research

\section{REFERENCES}

[1] H. Lin, L. Wang, and R. Kong, "Energy Efficient Clustering Protocol for Large-Scale Sensor Networks, “ IEEE Sensors Journal, vol. 15, no. 12, pp. 7150-7160, 2015.

[2] W.R. Heinzelman and A. Chandrakasan, "Energy-Efficient Communication protocol for Wireless Micro sensor Networks", Proc. 33rd Hawaii International Conference on System Sciences, 2000.

[3] A. Manjeshwar and D.P. Agrawal, "TEEN: A Routing protocol for Enhanced Efficiency in Wireless Sensor Networks", Proc. 15th International Parallel and Distributed Processing Symposium, 2001.

[4] G. Smaragdakis and I. Matta, "SEP: A Stable Election protocol for clustered heterogeneous Wireless sensor Networks", Boston University Computer Science Department, 2004.
[5] W.R. Heinzelman, A. Chandrakasan, and H. Balakrishnan, "Energyefficient communication protocol for wireless microsensor networks," Proc. 33rd Hawaii International Conference on System Sciences, vol. 2, pp. 1-10, 2000.

[6] P. Zou and Y. Lin,"A data-aggregation scheme for WSN based optimal weight allocation", Journal of Networks, vol. 9, no. 1, pp. 100-107, 2014.

[7] M.J. Handy, M. Haase, and D. Timmermann, "Low energy adaptive clustering hierarchy with deterministic cluster-head selection," Proc. 4th International Workshop on Mobile and Wireless Communication Networks, 2002.

[8] F Farouk, R. Rizk, and F.W. Zaki, "Multi level stable and energy efficient clustering protocol in heterogeneous wireless sensor networks", IET Wireless Sensor Systems, vol. 4, issue 4, pp. 159-169, 2014.

[9] S. Lindsey and C. Raghavendra, "Pegasis: Power-efficient gathering in sensor information systems," Proc. IEEE Aerospace Conference, vol. 3, pp. 1125-1130, 2002.

[10] A. Manjeshwar and D. Agrawal, "Apteen: a hybrid protocol for efficient routing and comprehensive information retrieval in wireless sensor networks," Proc. International Parallel and Distributed Processing Symposium IPDPS, 2002.

[11] B. Li, W.J. Wang, Q.Y. Yin, H.X. Li, and R. Yang, ”An energyefficient geographic routing based on cooperative transmission in wireless sensor networks", Science China Information Science, vol. 56, issue 7, pp. 1-10, 2013.

[12] Y. Yu, R. Govindan, and D. Estrin, "Geographical and energy aware routing: a recursive data dissemination protocol for wireless sensor networks," UCLA Computer Science Department Technical Report UCLA/CSD-TR-01-0023, Tech. Rep., 2001.

[13] W. Ye, J. Heidemann, and D. Estrin, "An energy-efficient MAC protocol for wireless sensor networks," Proc. IEEE INFOCOM, vol. 3, pp. 1567-1576, 2002.

[14] Y. Gao,"Wireless Sensor Network Adaptive Energy Optimization Clustering Algorithm based on Routing Rules", Journal of Networks, vol. 9, no. 7, pp. 1935-1940, 2014.

[15] A. El-Hoiydi and J.-D. Decotignie, "WiseMAC: An Ultra Low Power MAC Protocol for Multi-hop Wireless Sensor Networks." Proc. ALGOSENSORS 2004, pp. 18-31, 2004.

[17] K. Bellingham, "Method for irrigation scheduling based on soil moisture data acquisition", United States Committee on Irrigation and Drainage, 2009.

[18] X. Shu-ming, W. Liang-Min, Q. Xiao-qian, and Z. Yong-Zhao, "Application research of WSN in precise agriculture irrigation", Environmental Science and Information Application Technology, 2009. ESIAT 2009. International Conference on, vol. 2, pp. 297-300, 2009. 